\title{
From S-M-L-XL to Mass Customization Case Study: External Ankle Sprain Protection with Exo-L
}

\author{
Johan F.M.MOLENBROEK ${ }^{\star a}$, Marcel FLEUREN ${ }^{b}$, GertJan KLEIN RENSINK ${ }^{c}$ \\ ${ }^{a}$ Delft University of Technology, Delft, Netherlands; \\ ${ }^{b}$ Exo Ligament, Delft, Netherlands; \\ ${ }^{c}$ Erasmus MC, Rotterdam, Netherlands \\ http://dx.doi.org/10.15221/13.173
}

\begin{abstract}
This paper presents the conducted research and development when creating Exo-L: 'A bio inspired external ligament that effectively supports the ankle, without reducing the feeling of comfort and flexibility'. It uncovers some of the early research and the patented technical proof of principal, as well as a glimpse of the roadmap to Exo-L's market introduction. It will be discussed how a customized product proves to be superior compared to an universal set of products in certain situations, and how the current state of technology enables customization on a large scale.
\end{abstract}

An ankle sprain injury occurs when high impact forces make the foot rotate beyond its normal range of motion. In addition to adequate training, an external support helps to prevent these injuries from happening. The similarity between the evolved principles of the foot's ligaments (biomimicry) was guiding the analysis and concept development process of Exo-L and a solution was found that supports the relative motion between two rigid parts (the foot and the lower leg) by an external ligament means.

It was recognized that most previously available ankle braces rely on fixing some universally sized components tightly around the ankle region and therefor reducing the feeling of freedom, which is a key element for performance. Exo-L transfers forces from a special attachment feature on the shoe to the lower leg by introducing a perfectly fitting half open clip, allowing pressure to be exerted onto the malleoli. Exo-L is tailor made by 3D-scanning the user's ankle and subsequent 3D-printing of the product.

Keywords: Sprain, sport, injury, 3D anthropometry, ankle, external ligament, Exo-L

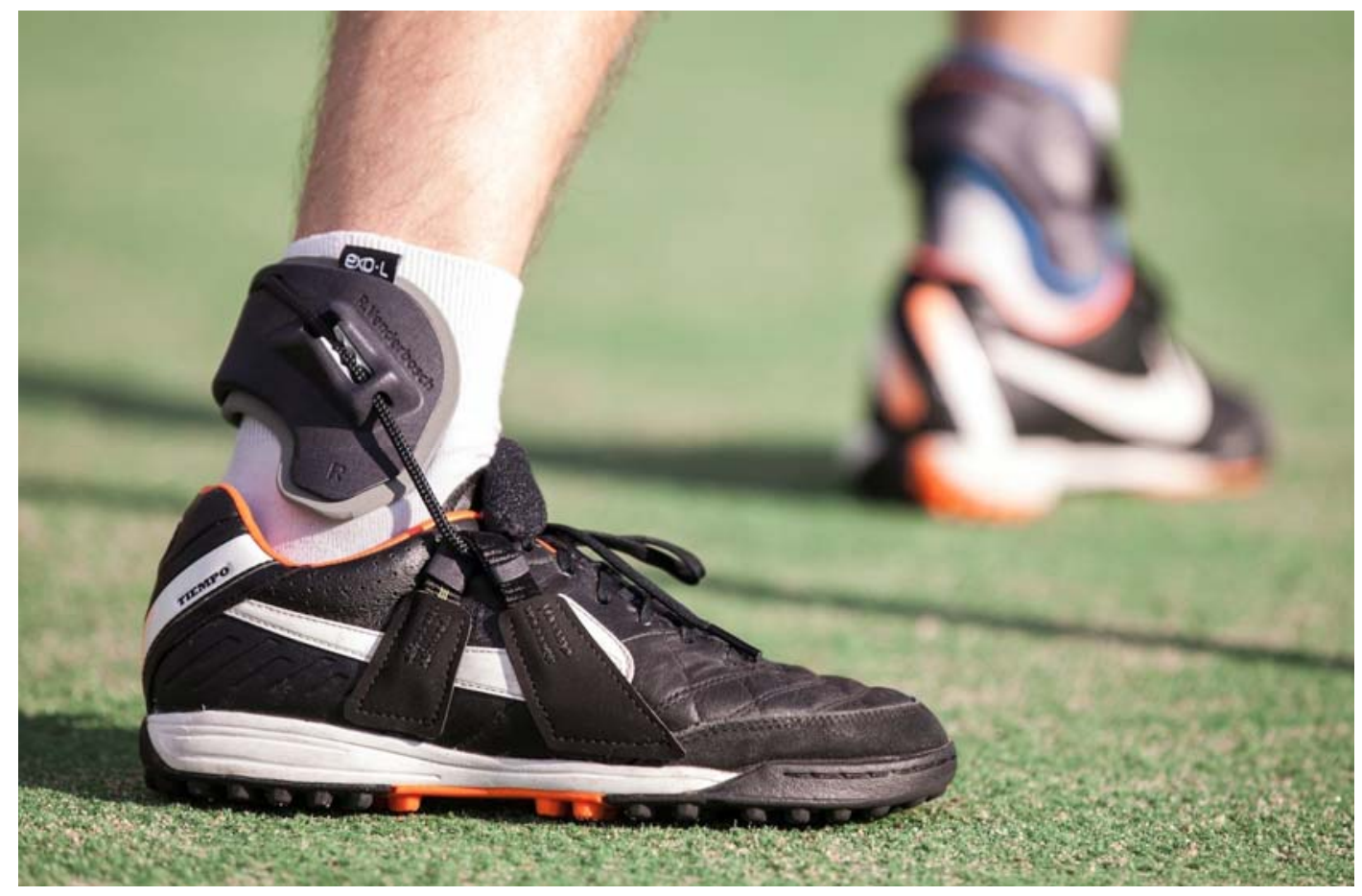

Figure 1. Exo-L: a customized external ligament. 


\section{Introduction}

\subsection{Explorative case study}

This paper presents an explorative case study to show how an innovation focused on biomimicry and mass-customization is developed within the context of scientific research, industrial design and entrepreneurship.

The faculty of Industrial Design Engineering (Delft University of Technology) has 40 years' experience in ergonomics and anthropometry. Target groups have been elderly, children, students, disabled and (adapted) sports in relation to a large variety of applications.

The second author was bachelor and master student at this faculty. During his final thesis project, under supervision of the first and last author, he developed an external ligament concept to prevent ankle sprains (Exo-L). After acquiring his MSc title, the company Exo Ligament was founded to further develop the invention and introduce a new product category to the market.

\subsection{Ankle sprains}

The ankle sprain injury is a worldwide problem and well known to most people $[1,2,3,4,13]$. It is also denoted as ankle roll or inversion trauma. A serious ankle sprain immobilizes people for one to six weeks at best, causing athletes to miss practices, competition matches and non-sports related activities. The injury mostly happens during sports involving running, cutting, jumping or physical contact.

$80 \%$ of the ankle sprains is the result of an unfortunate landing of the foot in an inverted position, being the result of stepping on an uneven surface or landing after being disoriented. The inversion trauma happens by excessive exo-rotation of the lower leg. An additional component of plantar flexion increases the chances of stretching ligaments. This movement is illustrated in Figure 2.

In the Netherlands, including 16 Million citizens, a total of 600.000 people suffer from the injury each year and multiple studies have identified the ankle sprain to be the most severe injury in multiple sports.
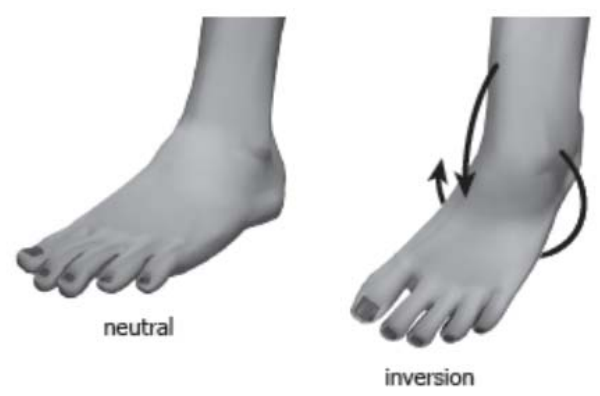

Figure 2. Inversion movement of the left foot.

\subsection{External ankle support}

Research has indicated that external ankle supports and tape both reduce the risk of ankle injuries in sports $[5,6,7,8]$. However, athletes often reject them as a preventive measure due to the introduced discomfort and inflexibility.

Figure 3 on the next page illustrates how a pilot group of athletes distributed different braces on a 'Protective' versus 'Sporty' scale. It can be seen that effective support of the ankle comes in most cases with reduced comfort. This is due to the braces becoming more bulky and motion restrictive, thereby exerting irritating pressure onto the skin, and taping being inconvenient for frequent users due to high recurring costs and skin irritation. Figure 3 also illustrates the authors' aim of introducing a new means of ankle sprain prevention that provides effective protection as well as an optimal feeling of freedom. 


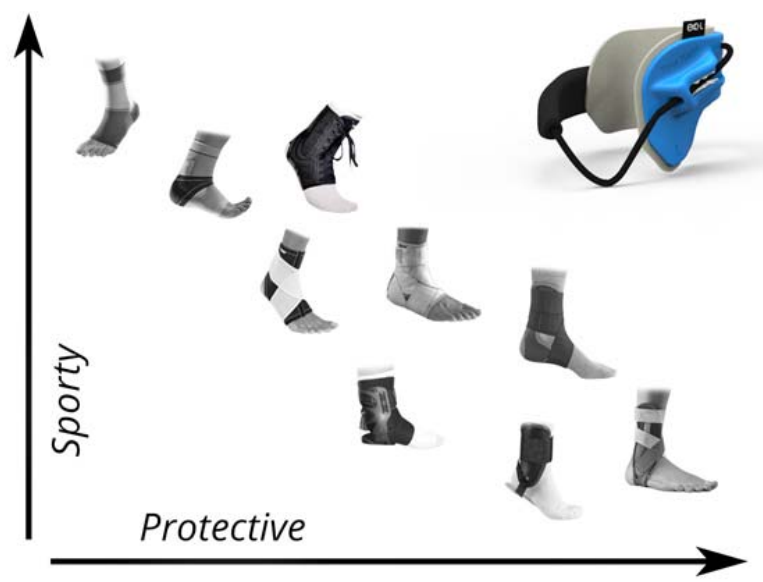

Figure 3. Distribution of ankle braces on a 'Protective' vs. 'Sporty' scale.

\section{Case Presentation}

\subsection{The creation of an external ligament}

The introduction above only briefly mentions the ankle sprain injury. This paragraph therefore starts with a more detailed explanation of the analyzed anatomical structures, which was a key element in the design process. Similarities between anatomical principles and the presented embodiment of Exo-L at the end of this chapter will be clear, underpinning it as a biomimicry design example.

\subsubsection{Analysis of the (functional) anatomy}

The lower leg consists of only two bones, the fibula and tibia. By contrast, the foot consists of 32 small bones whose interconnections enable its complex movements. At the places where different bones articulate (connect) a joint is formed and the bone surfaces are covered with a layer of articular hyaline cartilage. This offers a firm, smooth and relatively friction-free layer to facilitate joint movement. The location and names of the different bones are illustrated in Figure 4.

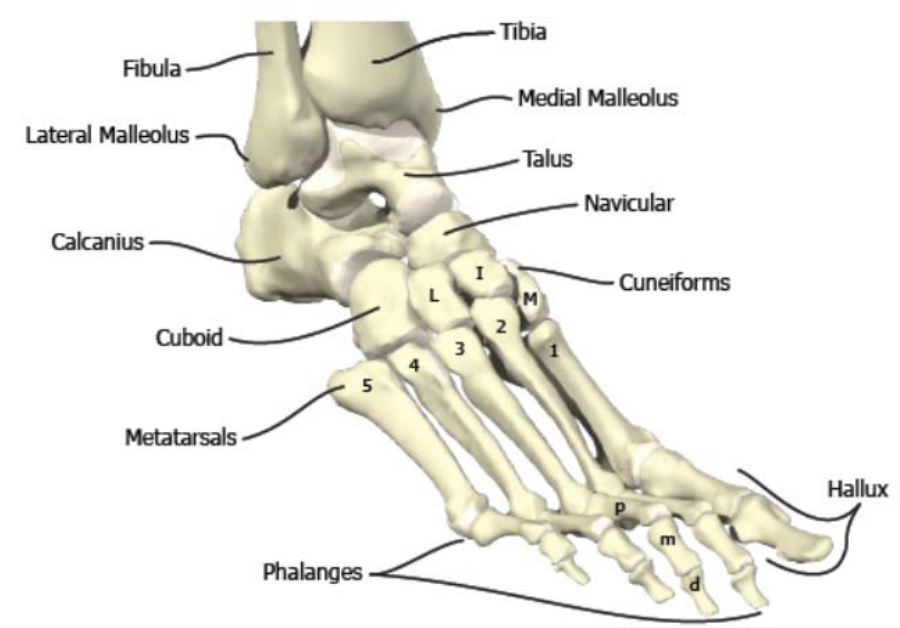

Figure 4. Bones in the foot and ankle region. Medial-lateral view of the right foot. 
Ligaments are connected to bones and serve to restrict the motion of a joint and ensure the integrity of the bony system. Ligaments are made of a visco-elastic, fibrous tissue. They show a form of relaxation (creep) under tensile forces, and the ligaments return to their original shape when the tension is removed. In the foot, a total of 108 ligaments can be found.

When too large a force is applied, the ligaments get stretched or teared. When an inversion trauma occurs the anterior talo-fibular ligament is in most cases damaged first, followed by the calcaneo-fibular ligament. Both ligaments can be found at the lateral side (outside) of the foot and are illustrated in Figure 5.

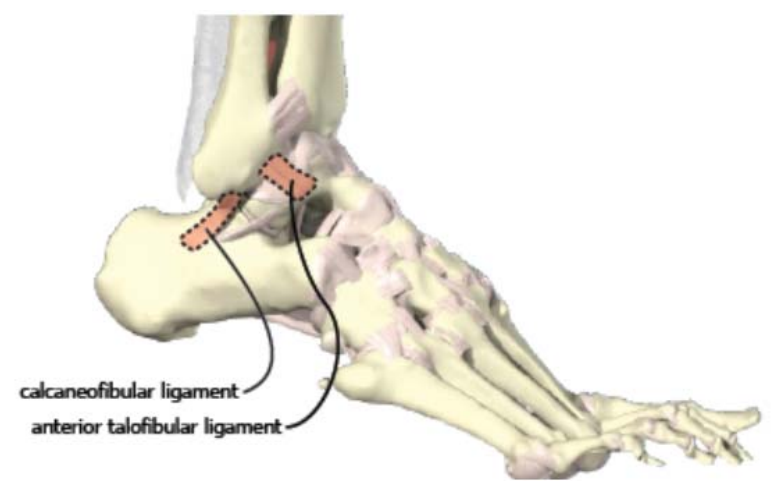

Figure 5. The ligaments that get damaged first when an inversion trauma occurs.

It is most easy to understand the function of the ligaments when the foot is represented as one single unit moving relative to the lower leg. Two rigid parts, the leg and the foot, move away from each other during activity. The ligaments are connected to both these parts (anchor points on the bones) and try to restrict this motion together with the evertor muscles (applying a counter effect to inversion). The direction and engagement points of their injury preventive forces are illustrated in Figure 5.

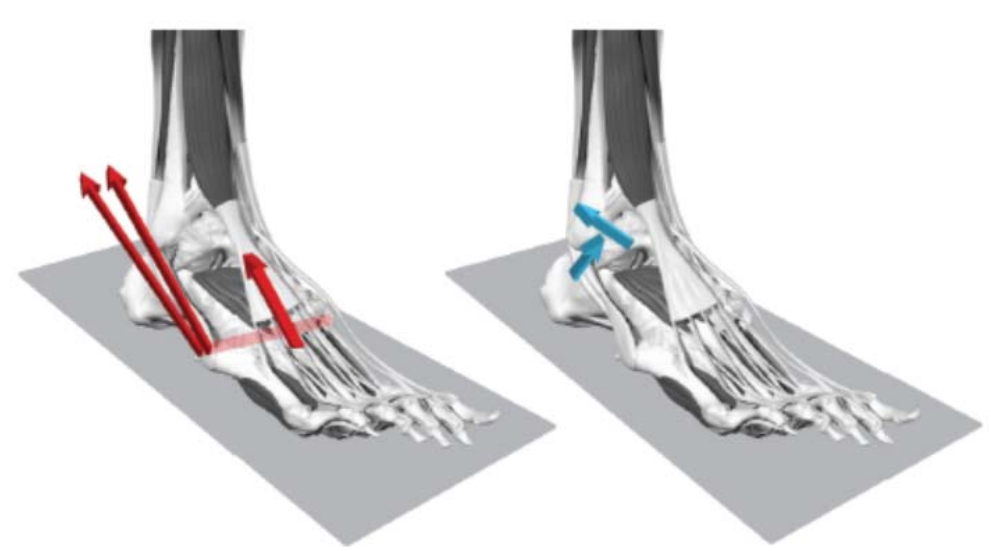

Figure 6. The direction and engagement points of evertor muscles (left) and lateral ligaments (right).

\subsubsection{Concept development}

It was investigated that, in analogy with the anterior talo-fibular, the force of an external ankle support can effectively be transferred from the foot to the lower leg when directed from marker I to marker B (Figure 7). In that case the shoe, as well as the extruding malleoli (bony ankle parts) can be used as anchor points. 


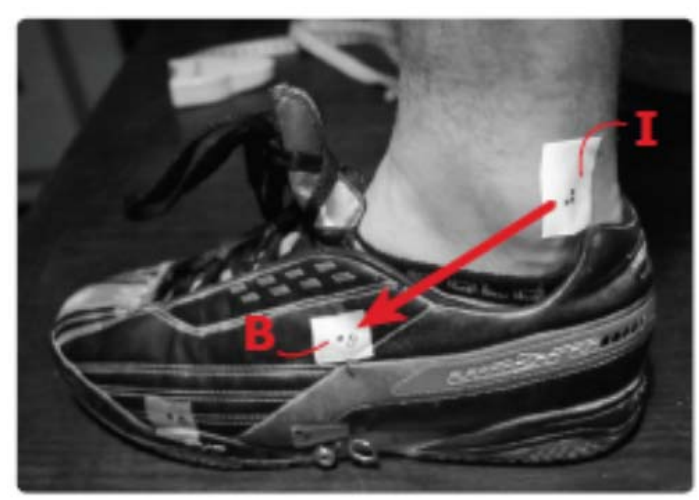

Fig. 7. Markers I and B on the lateral side of the leg and foot.

The data about the distance change from marker I to marker B between a neutral and supinated (s-n), pronated ( $p-n)$ and inverted (i-n) position of the foot was initially measured within a group of 20 people and is summarized in Table 1 . Since the distance between marker I and marker B tends to increase when an inversion (sprain) movement occurs, it was reasoned that restricting an excessive increase of distance between these points is also limiting the risk for injuries. This is the thought that eventually evolved into the Exo-L concept.

Table 1. Difference in I-B distance between neutral and rotated positions.

\begin{tabular}{lrrrrr}
\hline & $5-\mathrm{n}$ & $\mathrm{p}-\mathrm{n}$ & $\mathrm{i}-\mathrm{n}$ & $\mathrm{i}-\mathrm{s}$ & $\mathrm{i}-\mathrm{p}$ \\
\hline Average & 12 & 9 & 20 & 8 & 11 \\
Maximum & 24 & 10 & 29 & 12 & 20 \\
Minimum & 8 & 5 & 14 & 4 & 4 \\
\hline
\end{tabular}

The measurements A - D, indicated in Figure 8, have been recorded in researches amongst thousands of soldiers $[9,10]$. However, to effectively create anchor points as mentioned earlier in this section, additional anthropometric data is relevant and was recorded within a small target group. This includes the distance of the medial and lateral malleoli with respect to the heel and the distribution of the total malleolar breadth. They are indicated by measurements 1 - 4 in Figure 8.

There exists a huge variation in body measurements between people. Furthermore, the different measurements are not all correlated with each other. Having a high medial malleolus for example does not necessarily mean the lateral malleolus will be high as well. The authors' experience with the huge variation in body dimensions resulted into the early thoughts that a custom fitted product has many advantages in this case.
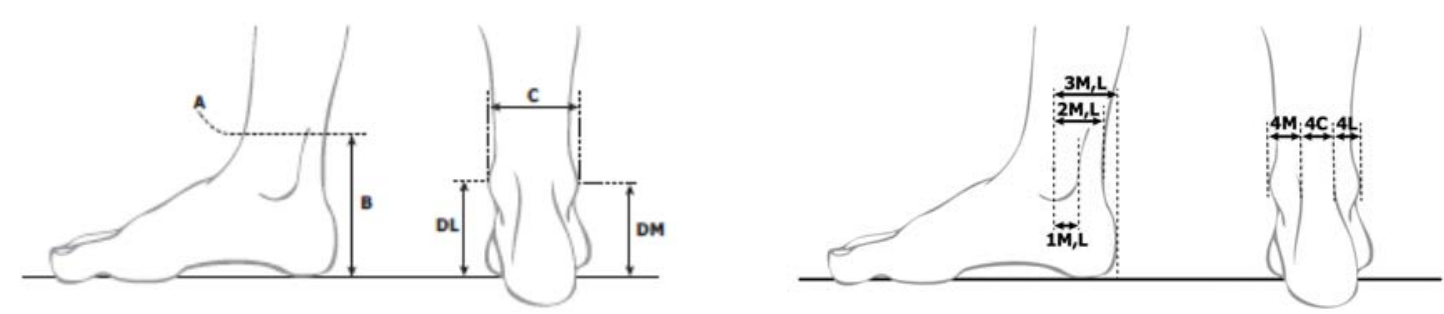

Fig. 8. Anthropometric measurements. 


\subsubsection{Proof of principle}

The problem analysis summary in previous paragraphs shows aspects that firstly had to be united in a working prototype. Many trials and force tests were performed in a laboratory setting (Figure 9) before the analogy with the anterior talofibular ligament was transformed into an external ligament.

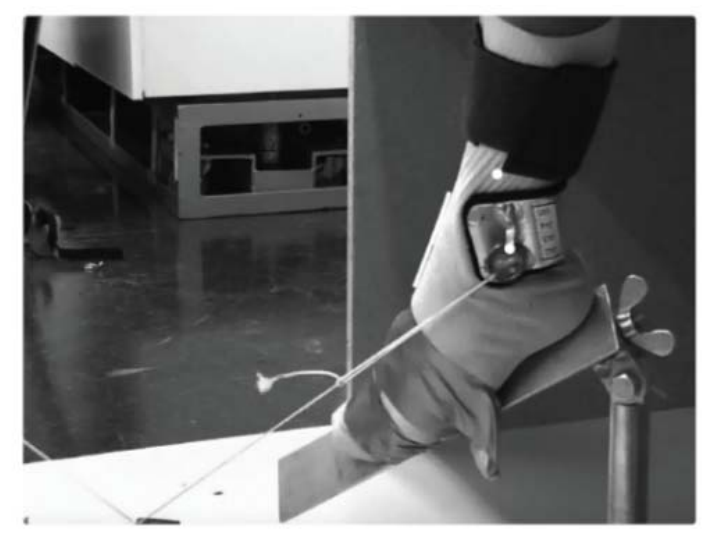

Figure 9. Testing of principle in laboratory.

Eventually a 3D-scan of the graduating student's ankle was used to produce the first (3D printed) prototype. A clamping mechanism was glued inside a recess on the lateral side of the clip and the prototype was finished with a band and padding material, illustrated in Figure 10. The possibilities of sewing an attachment feature to the shoe were investigated as well and five athletes and their medical advisors were found to test the next few prototypes. The promising reactions of users have since then been the main motivation for further development.
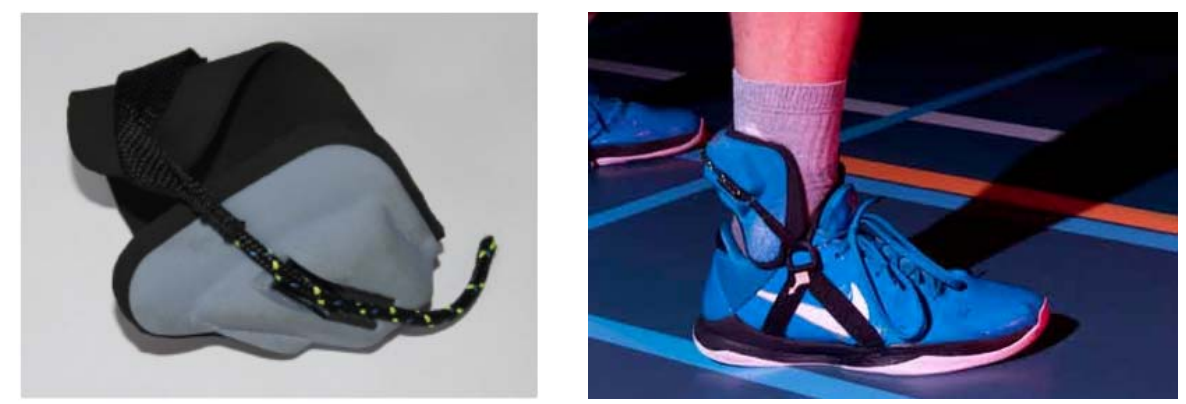

Figure 10. First prototype and user test.

\subsubsection{Patent application}

Patent databases have been used to look for similar technical principles. The designed external ligament was found to be new compared to the current state of art and during the early development stages a new patent application was recorded in a patent application on June 10th 2011 (figure 11). The Dutch patent has been granted at the time of writing and international expansion has been filed in other European countries and overseas.

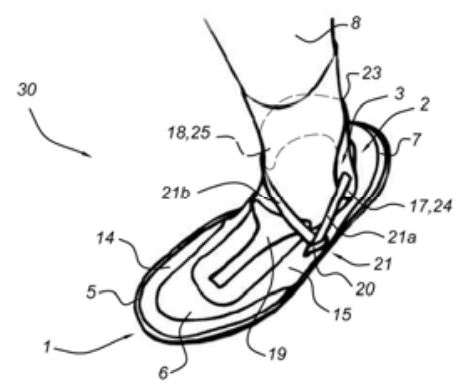

Figure 11. Patent drawing. 


\subsection{Product development}

The concept for an external ligament was initially developed during a thesis project within the Delft University of Technology and the Erasmus Medical Centre. However, before officially introducing a product like Exo-L to the market, further investigation, embodiment, and logistic optimization are asked for. This section describes what steps took place during the startup phase of a company called Exo Ligament, which was founded to valorization the invention.

\subsubsection{Universal vs. custom}

In an early phase several project were initiated to further investigate the most accurate fit of Exo-L for many people at the same time. The customization of every single Exo-L with 3D scanning and 3D-printing was compared to alternatives using a set of predefined sizes (figure 12). In addition, the use of thermoplastic materials which allow ad hoc adjustment was investigated as well.
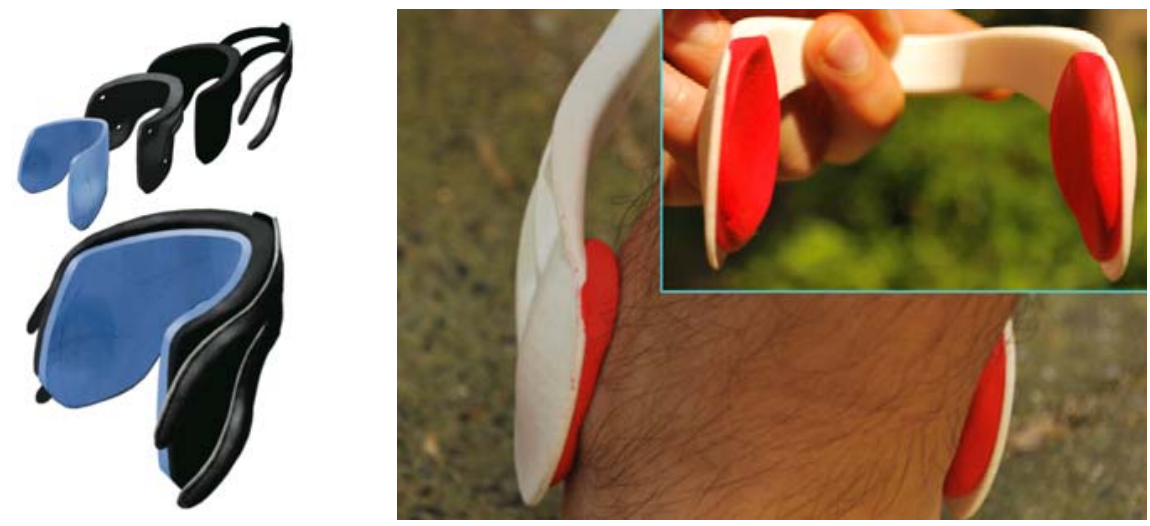

Figure 12. Patent drawing.

By doing continuous user tests it was discovered that, even when having 10 of 20 fixed sizes using thermoplastic materials instead of a simple $\mathrm{S}, \mathrm{M}, \mathrm{L}, \mathrm{XL}$ variation, the product would become bulky as a epiphenomenon of universality. An (3D-printed) exact fit to the user's body, being the most sporty and elegant alternative, proved to have added significant value to the majority of users.

\subsubsection{Optimization, Documentation en Certification.}

The shape of Exo-L and the exact way it is connected to the user's body has revolutionized during the past two years. A total of over 100 prototypes, all featuring incremental structural changes, have been made to test every aspect of the product. Furthermore, since Exo-L is a medical product, it was subjected to many obligatory tests. This resulted in a technical documentation that describes the product details and production process. Figure 13 illustrates some of the testing procedures that took place in cooperation with the Delft University of Technology.
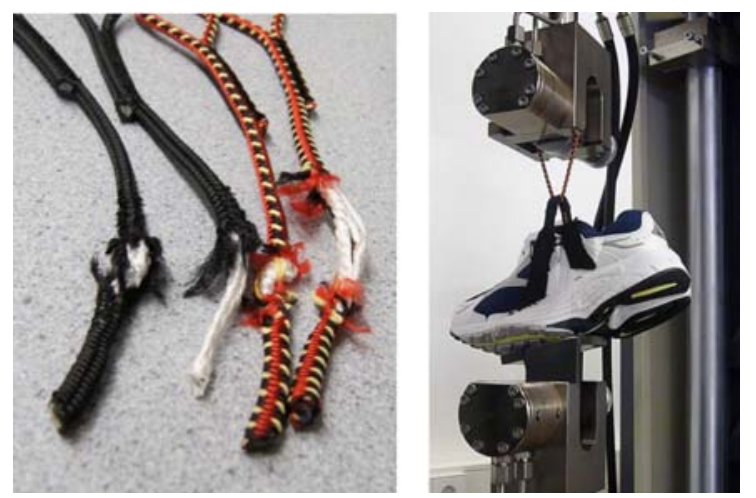

Figure 13. Product testing procedures. 
The process of acquiring 3D-data and subsequently using that data to construct computer models that can be 3D-printed started as a time-consuming task. However, the necessary optimization steps for making mass customization possible have been taken by the company Exo Ligament. The system which acquired data through a 3D-scanner in shops or at physiotherapist practices is the first step in that process. When a customer then converts his scan into an order, one of the developers uses a refined parametric model generator that reduced the computer modeling time significantly. Figure 14 shows an impression of these two steps in the process.
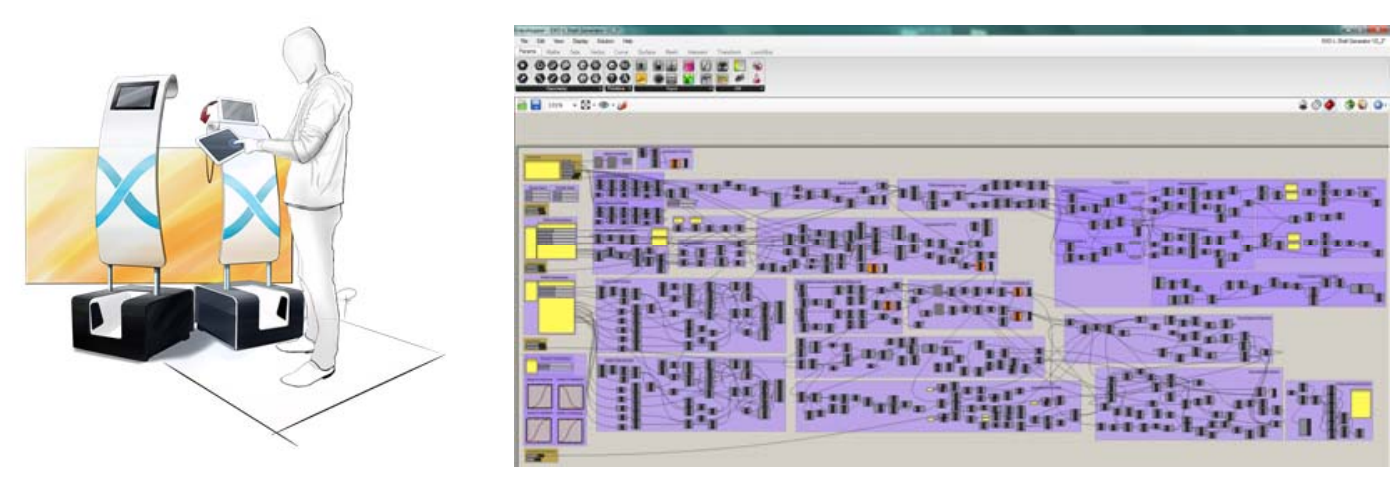

Figure 14. 3D-scanner in shops \& Parametric model chart.

\subsubsection{User pilots}

During the development of Exo-L three major subsequent pilots have been performed. The product has thus been extensively tested in every phase of the development process, which was seen by the authors as the most valuable input during the development process. The first two pilots were mainly executed to gather feedback for further optimization, while the last pilot served mainly for validation and included 70 athletes practicing 8 different sports. $93 \%$ of the participants experience an increased feeling of support and $84 \%$ of them were enthusiastic about the increased feeling of freedom. These results have been a driver for officially introducing the product to the market.

\section{Conclusions}

This case study demonstrates the development of a product that fits the individual customer (Exo-L). The importance for the readers of this paper is the understanding of methods, techniques and efforts necessary to develop such a product from scratch to market implementation. This process is expected by the authors to be the same for similar mass customization concepts.

The foundation of Exo-L's design is closely related to biomimicry. In that light the external ligament of Exo-L imitates the human anatomy in two ways:

1) It respects the functional anatomy of ligaments, since it only inhibits unnatural and excessive movements by allowing the transfer of injury causing forces without the need for strapping something tight around the foot and lower leg. Only when the foot is excessively inverted the external ligament of Exo-L will be tightened. The product is easy to fit and improves flexibility and training of the ankle joint.

2) It respects the endless inter- and intra individual variation as can be observed in human anatomy. Not two ankles are identical, not even in identical twins. An accurate fit to the users body is achieved by the use of 3D-scanning and 3D-printing.

After successfully completing several pilots, the company Exo Ligament is currently introducing their product to the market. This is an early example application where mass customization is chosen and preferred over the use of a standard S, M, L, XL confection product. A division into 4 or even 10 variations is unable to serve the mentioned purposes. State of the art technologies like parametric modeling and a creative approach of the market make mass customization feasible. Exo Ligament shows how a high-end custom made 3D-printed product can be delivered to the customer within a week after acquiring 3D-data of the user's body. 


\section{References}

1. Fong DTP Chan YY, Mok KM, Yung PSH, Chan KM: Understanding acute ankle ligamentous sprain injury. Sports medicine, arhroscopy, rehabilitation, therapy \& technology, issue 1 (2009).

2. Ankle braces to prevent injuries, Amsterdam, NL (2010): Eurosafe.

3. Ivins D: Acute ankle sprain: an update. American family physician, issue 74 (2006), p1614-1720.

4. Waterman BR, Owens BD, Davey S, Zacchilli MA, Belmont PJ: The Epidemiology of Ankle Sprains in the United States, Volume 92, issue 13 (2010), p2279-2284.

5. Barnett $\mathrm{CH}$, Napier $\mathrm{JH}$ : The axis of rotation at the ankle joint in man. Journal of anatomy, issue 87 (1953), p207-213.

6. Ankle tape to prevent injuries, Amsterdam, NL (2010): Eurosafe.

7. Gross MT, Liu HY: The role of ankle bracing for prevention of ankle sprain injuries. Journal of orthopaedic \& sports physical therapy, issue 33 (2003, p572-577

8. Dizon JMR, Reyes JJB: A systematic review on the effectiveness of external ankle supports in the prevention of inversion ankle sprains among elite and recreational players. Journal of Science and medicine in sport, issue 13 (2010, p309-317.

9. Anthropometric source book volume II, a handbook of anthropometric data. Yellow Springs, U.S. (1978): NASA scientific and technical information office.

10. Parham KR, Gordon CC, Bensel CK: Anthropometry of the foot and lower leg of U.S. army soldiers. Natick, U.S. (1992):

11. Consumer Safety Institution www.veiligheid.nl

12. Fleuren,M., Exo-I MSc-Report. Faculty Industrial Design Engineering, TUDelft, 2011.13

13. Kemler E, van de Port I, Backx F, van Dijk CN. A systematic review on the treatment of acute ankle sprain: brace versus other functional treatment types. Sports Med. 2011 Mar 1;41(3):185-97 